ARTICLE

\title{
Quantum frequency doubling in the topological insulator $\mathrm{Bi}_{2} \mathrm{Se}_{3}$
}

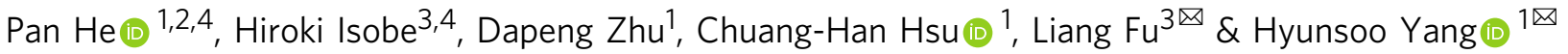

The nonlinear Hall effect due to Berry curvature dipole (BCD) induces frequency doubling, which was recently observed in time-reversal-invariant materials. Here we report novel electric frequency doubling in the absence of $B C D$ on a surface of the topological insulator $\mathrm{Bi}_{2} \mathrm{Se}_{3}$ under zero magnetic field. We observe that the frequency-doubling voltage transverse to the applied ac current shows a threefold rotational symmetry, whereas it forbids BCD. One of the mechanisms compatible with the symmetry is skew scattering, arising from the inherent chirality of the topological surface state. We introduce the Berry curvature triple, a high-order moment of the Berry curvature, to explain skew scattering under the threefold rotational symmetry. Our work paves the way to obtain a giant second-order nonlinear electric effect in high mobility quantum materials, as the skew scattering surpasses other mechanisms in the clean limit.

\footnotetext{
${ }^{1}$ Department of Electrical and Computer Engineering, National University of Singapore, Singapore 117576, Singapore. ${ }^{2}$ Institute for Nanoelectronic devices and Quantum computing, Fudan University, Shanghai 200433, China. ${ }^{3}$ Department of Physics, Massachusetts Institute of Technology, Cambridge, MA 02139, USA. ${ }^{4}$ These authors contributed equally: Pan He, Hiroki Isobe. ${ }^{凶}$ email: liangfu@mit.edu; eleyang@nus.edu.sg
} 
T he Hall effect, the generation of voltage transverse to an electric current and a magnetic field, and the anomalous Hall effect (AHE) in magnetic materials ${ }^{1}$ require timereversal symmetry breaking. These effects refer to a transverse electric response in the linear region, where the Hall voltage $V_{y}$ scales linearly with the longitudinal current $I_{x}$. The second-order (nonlinear) Hall effect, in which $V_{y}$ depends quadratically on $I_{x}$, has attracted attention in condensed matter physics ${ }^{2-4}$. A quantum origin of the nonlinear Hall effect in time-reversal-invariant materials is the Berry curvature dipole $(B C D)^{3}$. The nonlinear Hall effect due to the BCD was observed recently in bilayer and few-layer $\mathrm{WTe}_{2}{ }^{5,6}$. The $\mathrm{BCD}$ generates an effective magnetic field in a stationary state, thus leading to the nonlinear Hall effect ${ }^{3}$. Electrical second-harmonic generation (SHG), including the nonlinear Hall effect, can exist only when a system lacks inversion symmetry ${ }^{7-9}$. Despite growing interest of $\mathrm{BCD}^{10-14}$, it is subject to strict crystal symmetry restrictions and vanishes in certain crystals even without inversion symmetry ${ }^{3}$, while second-order response is still allowed. Therefore, a search for electrical SHG independent of the BCD is desirable.

Inversion symmetry is absent in low-symmetry crystals (such as $\left.\mathrm{WTe}_{2}{ }^{5,6,10}\right)$, and on a surface or an interface. However, the electrical SHG has not explored in surface/interface systems with time-reversal symmetry. Three-dimensional (3D) topological insulators (TIs) have attracted great interest due to the topological surface state (TSS) with spin-momentum locking ${ }^{15-17}$ for applications in spintronics and quantum computing ${ }^{18-20}$. With an inversion-symmetric bulk, $3 \mathrm{D}$ TIs such as $\mathrm{Bi}_{2} \mathrm{Se}_{3}, \mathrm{Bi}_{2} \mathrm{Te}_{3}$, and $\mathrm{Sb}_{2} \mathrm{Te}_{3}$ host electrical SHG only on the surfaces. Furthermore, threefold rotational symmetry of the TI surface in Fig. 1a forces a $\mathrm{BCD}$ to vanish (Fig. 1b) ${ }^{3}$; thus, the BCD-induced nonlinear Hall effect is not allowed. In addition to the intrinsic contribution by a $\mathrm{BCD}$, extrinsic effects arising from impurity or phonon scatterings, as intensively studied in $\mathrm{AHE}^{1}$, are yet to be well sorted out for nonlinear effects. 3D TIs are ideal platforms in searching for extrinsic electrical SHG in the absence of a BCD. While recent theoretical studies addressed extrinsic mechanisms ${ }^{21-24}$, an experimental observation of extrinsic contributions to the electrical SHG has not been reported.

In this work, we show the observation of electrical SHG in the $3 \mathrm{D} \mathrm{TI} \mathrm{Bi}_{2} \mathrm{Se}_{3}$ with time-reversal symmetry. The transverse voltage response depends quadratically on the applied current in the nonmagnetic $\mathrm{Bi}_{2} \mathrm{Se}_{3}$ films under zero magnetic field. The observed second-order response follows a threefold rotational symmetry on the surface of $\mathrm{Bi}_{2} \mathrm{Se}_{3}$. Notably, the symmetry excludes a BCD, which distinguishes the mechanism for electrical SHG from the previous studies 5,6 . We consider our observation arising dominantly from skew scattering in the TSS with its inherently chiral wave function. Instead of a BCD, we introduce the Berry curvature triple, which quantifies the moment of the Berry curvature under the threefold rotational symmetry. The skew scattering mechanism applies to a much wider class of noncentrosymmetric materials as broken inversion is the only symmetry constraint unlike the BCD.

\section{Results}

Observation of electric SHG. High-quality $\mathrm{Bi}_{2} \mathrm{Se}_{3}$ films were grown on $\mathrm{Al}_{2} \mathrm{O}_{3}$ (0001) substrates in a molecular beam epitaxy system. The first quintuple layer (QL) of $\mathrm{Bi}_{2} \mathrm{Se}_{3}$ is completely relaxed by van der Waals bonds ${ }^{25}$. In addition, the lattice constant of $\mathrm{Bi}_{2} \mathrm{Se}_{3}$ film relaxes to its bulk value, implying the absence of strain from the substrate ${ }^{25}$. Thus, the induction of BCD via a

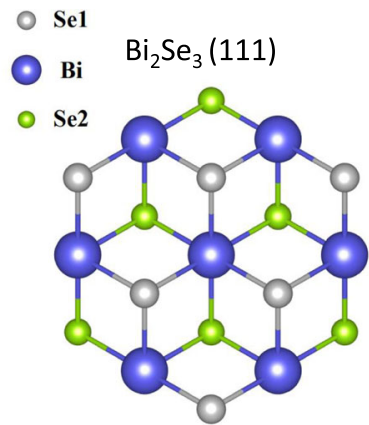

C
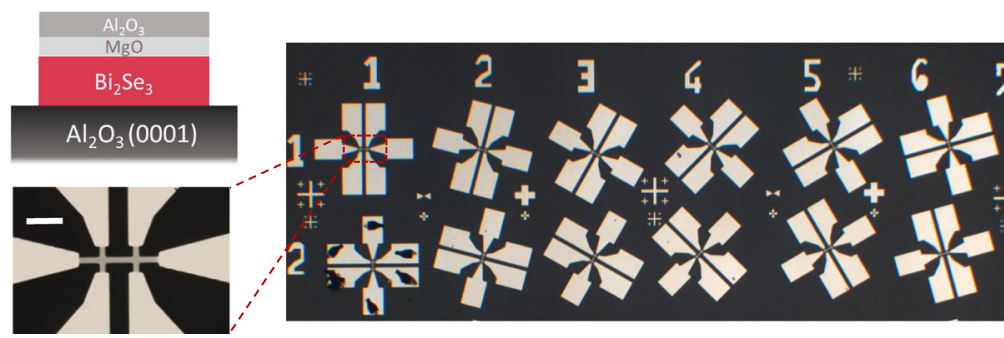

b

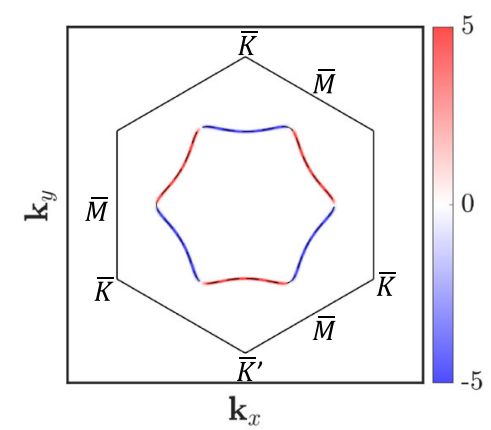

$\mathbf{k}_{x}$

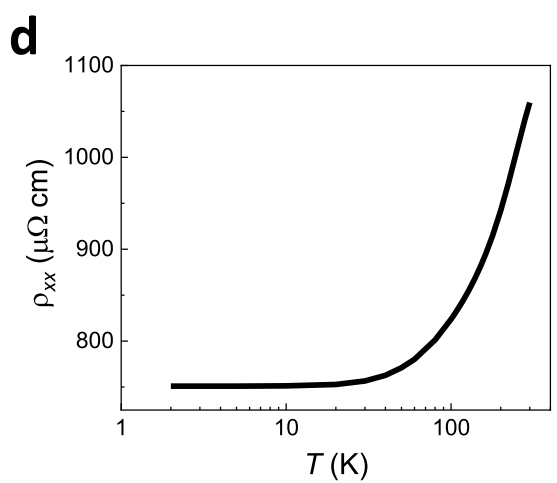

e

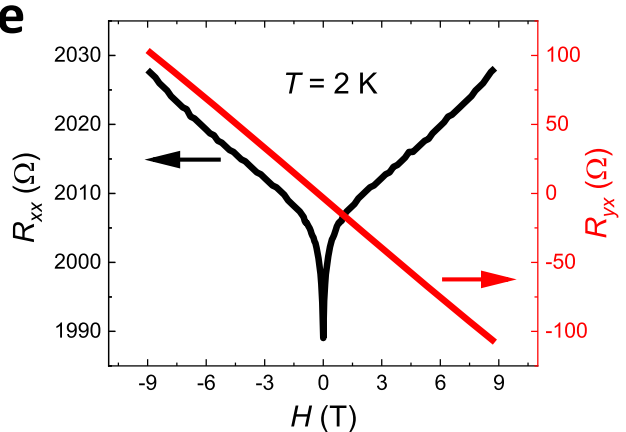

Fig. 1 Crystal structure, Berry curvature distribution, and characterizations of $\mathbf{B i}_{\mathbf{2}} \mathbf{S e}_{\mathbf{3}}$. a Crystal structure of $\mathrm{Bi}_{2} \mathrm{Se}_{3}$. From top view along the $z$-direction of $\mathrm{Bi}_{2} \mathrm{Se}_{3}$ (111) surface, the triangle lattice in one quintuple layer has three different positions, denoted as Se1, Bi, and Se2. b Schematic of Berry curvature distribution on the Fermi surface (FS) of TSS. The colored hexagon represents the hexagonally warped FS. The blue and red color contours indicate the negative and positive Berry curvature in an arbitrary unit. The black hexagon represents the surface Brillouin zone. c Schematic of the sample structure and optical image of Hall bar devices with the current channel along different directions. The inset shows magnified view of a device. The scale bar is $100 \mu \mathrm{m}$. d Temperature dependence of the longitudinal resistivity $\left(\rho_{\mathrm{xx}}\right)$ under zero magnetic field in $20 \mathrm{QL} \mathrm{Bi}_{2} \mathrm{Se}_{3}$. e Magnetic field dependence of the longitudinal resistance $\left(R_{x x}\right)$ and Hall resistance $\left(R_{y x}\right)$ at $2 \mathrm{~K}$ in $20 \mathrm{QL} \mathrm{Bi}_{2} \mathrm{Se}_{3}$. 

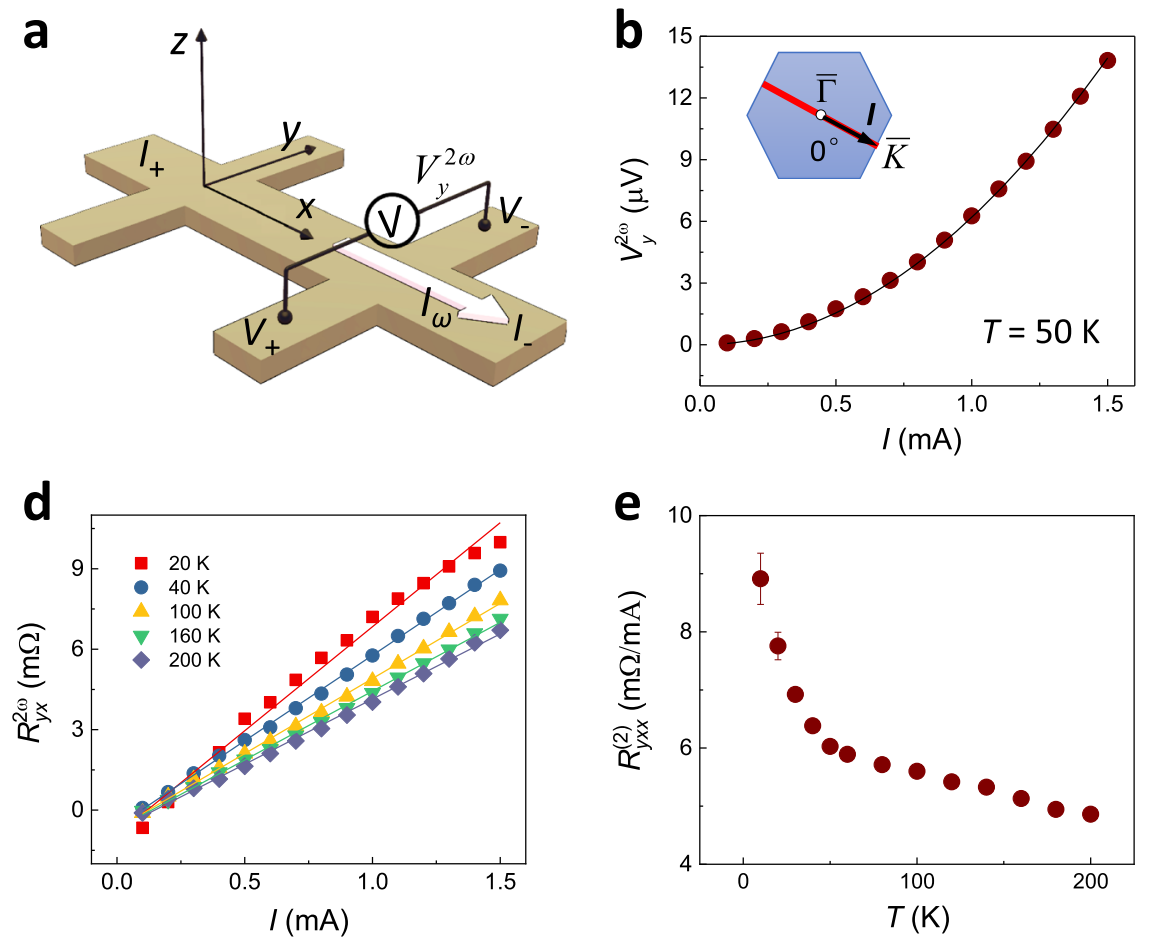

Fig. 2 Observation of electric SHG under zero magnetic field in $\mathbf{B i}_{\mathbf{2}} \mathbf{S} \mathbf{e}_{\mathbf{3}}$. $\mathbf{a}$ Schematic illustration of the nonlinear transport measurements in a Hall bar device using the second harmonic method. $\mathbf{b}$ The second harmonic transverse voltage $V_{y}^{2 \omega}$ versus ac current amplitude $/$ in 20 QL Bi $\mathrm{Se}_{3}$ at $50 \mathrm{~K}$. The solid line is a quadratic fit to the data. Blue hexagon in the inset represent the $\mathrm{FS}$ of $\mathrm{Bi}_{2} \mathrm{Se}_{3}$. Red line is along the $\bar{\Gamma} \bar{K}$ direction, and the black arrow denotes the current direction in k-space. c The second harmonic transverse resistance $R_{y x}^{2 \omega}$ scales linearly with $/$ at $50 \mathrm{~K}$. It changes sign with reversing the current direction and corresponding Hall probes. $\mathbf{d}$ The $R_{y x}^{2 \omega}(I)$ curves measured at different temperatures from 20 to $200 \mathrm{~K}$. e The slope of $R_{y x}^{2 \omega}(I)$ curves $\left(R_{y x x}^{(2)}\right)$ as a function of temperature. Error bars correspond to the standard error of linear fitting. $\mathbf{f}$ The measured carrier mobility $\mu$ as a function of temperature.

breaking the threefold rotational symmetry ${ }^{26}$ does not occur in $\mathrm{Bi}_{2} \mathrm{Se}_{3}$ films, as confirmed by our angle dependent transport measurements below. Multiple Hall bar devices with current channels along different crystalline directions (Fig. 1c) were fabricated. Figure 1d, e show the basic electrical characterization. The longitudinal resistivity $\rho$ (Fig. 1d) shows a typical metallic behavior and saturates below $\sim 30 \mathrm{~K}^{27,28}$. Figure 1e displays the longitudinal $R_{x x}$ and Hall $R_{y x}$ resistances as a function of an outof-plane magnetic field at $2 \mathrm{~K} . R_{x x}$ at the low field region exhibits the effect of weak anti-localization, indicative of $2 \mathrm{D}$ surface transports ${ }^{29}$. $R_{y x}$ depends linearly on the magnetic field, from which the $n$-type carrier density $n_{2 \mathrm{D}}$ is extracted to be $\sim 6.26 \times$ $10^{13} \mathrm{~cm}^{-2} . n_{2 \mathrm{D}}$ changes $<2.3 \%$ for temperature $(T)$ of $2<T<$ $300 \mathrm{~K}$.

To explore the nonlinear electric transport, we perform harmonic measurements using low-frequency lock-in techniques schematically shown in Fig. 2a. We apply the ac current $I_{x}(t)=$ Isin $\omega t$ along the $x$ direction and measure the voltage $V_{y}$ perpendicular to the current. Under time-reversal and threefold rotational symmetries, the transverse voltage response does not contain the linear contribution, leading to the expression

$$
V_{y}=R_{y x x}^{(2)} I_{x}^{2}
$$

which contains the SHG signal $V_{y}^{2 \omega}=\frac{1}{2} R_{y x x}^{(2)} I^{2} \sin (2 \omega t-\pi / 2)$. Note that the coefficient $R_{y x x}^{(2)}$ is proportional to the second-order conductivity $\sigma_{y x x}^{(2)}$ (see Supplementary Note 1 ), which can be finite in noncentrosymmetric materials ${ }^{3}$.

Figure $2 \mathrm{~b}$ shows the second harmonic transverse voltage under zero magnetic field in $20 \mathrm{QL} \mathrm{Bi}_{2} \mathrm{Se}_{3}$. Its quadratic dependence on the ac current $\left(V_{y}^{2 \omega} \propto I^{2}\right)$ reveals the electrical SHG from a timereversal-invariant $3 \mathrm{D}$ TI. Equivalently, the second harmonic transverse resistance defined as $R_{y x}^{2 \omega} \equiv V_{y}^{2 \omega} / I$ scales linearly with $I$ (Fig. 2c). Moreover, it changes the sign when we invert the current direction and the corresponding Hall probes (schematic in the inset of Fig. 2c). This is consistent with the second-order nature of nonlinear transport in Eq. (1). The electric SHG has little dependence on the input frequencies ranging from 9 to 263 $\mathrm{Hz}$ (see Supplementary Fig. 1).

Figure $2 \mathrm{~d}$ displays the $R_{y x}^{2 \omega}(I)$ data at different temperatures. The slope of $R_{y x}^{2 \omega}(I)$ (i.e. $\left.R_{y x x}^{(2)}\right)$ quantifies the magnitude of the electrical SHG. $R_{y x x}^{(2)}$ decreases gradually as temperature increases in Fig. 2e. In general, finite temperature affects the nonlinear electric transport through thermal smearing of the electron distribution function $f$ and the change of the electron scattering time $\tau$. Thermal smearing has little effect on the result as the Fermi energy is much higher than thermal energy $k_{B} T$ in our $\mathrm{Bi}_{2} \mathrm{Se}_{3}\left(k_{B}\right.$ : the Boltzmann constant). To reveal the effect of $\tau$, we depict the measured carrier mobility $\mu$ in Fig. $2 \mathrm{f}$. Both the SHG signal and mobility tend to decrease as temperature rises.

Angular dependence and scaling of nonlinear transport. To characterize the angular dependence of nonlinear electric transport, we measure various devices with the current applied along different crystal directions on $20 \mathrm{QL} \mathrm{Bi}_{2} \mathrm{Se}_{3}$ (Fig. 1c). The current direction is denoted by angle $\Theta$ with respect to the $\overline{\Gamma K}$ direction (i.e., $[-1,1,0]$ direction on $\mathrm{Bi}_{2} \mathrm{Se}_{3}$ (111) surface of the primitive lattice in real space) in Fig. 3. $R_{y x}^{2 \omega}$ shows the maximum value when the current direction is along $\overline{\Gamma K}$ (Fig. 2b, c), and decreases when the current is rotated $15^{\circ}$ away from $\overline{\Gamma K}$ in Fig. 3a. For $\Theta=$ 30 , i.e., with the ac current along the $\overline{\Gamma M}$ direction, $R_{y x}^{2 \omega}$ becomes vanishingly small (Fig. $3 \mathrm{~b}) . R_{y x}^{2 \omega}$ switches sign with a similar 
a
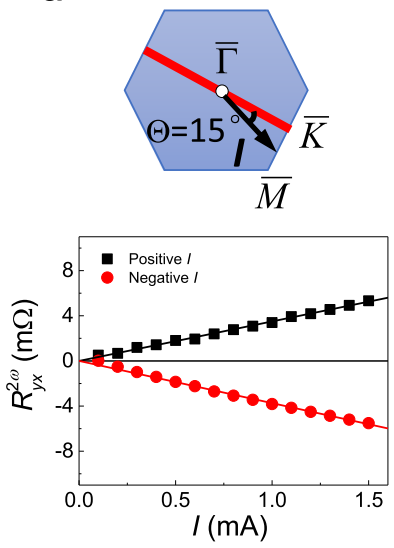

b
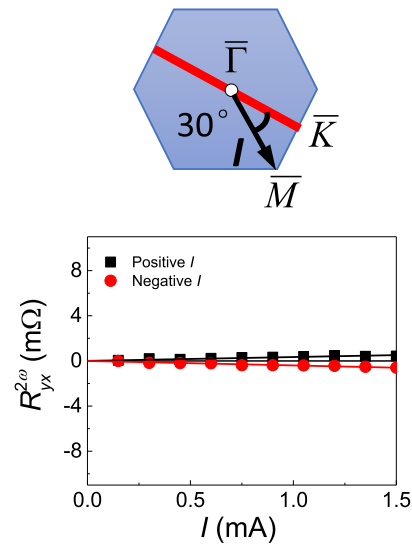

C
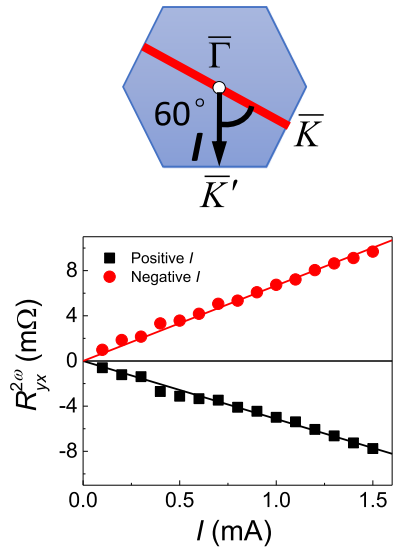

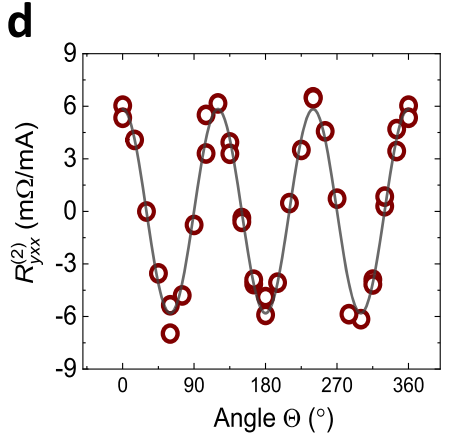

Fig. 3 Angular dependence of nonlinear transport in $\mathbf{B i}_{\mathbf{2}} \mathbf{S e}_{\mathbf{3}}$. The second-harmonic transverse resistance versus current for three typical current injection

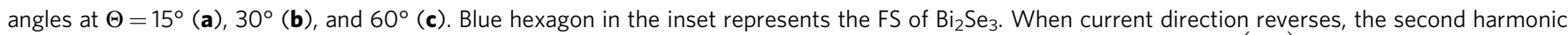
resistance changes sign in all current directions. The solid lines are linear fits to the data. $\mathbf{d}$ The slope of $R_{y x}^{2 \omega}(I)$ curves $\left(R_{y x x}^{(2)}\right)$ as a function of current direction. The data in panels a-d were collected at $T=50 \mathrm{~K}$.

magnitude when the current direction is rotated by $60^{\circ}$ from the $\overline{\Gamma K}$ to $\overline{\Gamma K}^{\prime}$ direction in Fig. 3c. The small non-symmetry of $R_{y x}^{2 \omega}(I)$ at the positive and negative current in Fig. $3 \mathrm{a}-\mathrm{c}$ can be due to misalignments of Hall bar. The electric SHG measured at 24 different directions is summarized in Fig. 3d, which shows the threefold angular dependence of $R_{y x x}^{(2)}$. The similar angular dependence is also observed in $10 \mathrm{QL} \mathrm{Bi}_{2} \mathrm{Se}_{3}$ (Supplementary Fig. 2). We emphasize that threefold rotational symmetric signal with sign change excludes the Joule heating effect as an origin, which is isotropic and generally leads to the third harmonic generation. The threefold symmetry also excludes a $\mathrm{BCD}$, while the helical spin texture ${ }^{30}$ and the Berry curvature ${ }^{31}$ (Fig. 1b) on the hexagonally warped Fermi surface (FS) of the TSS $^{32,33}$ share the same angular dependence. We note that the Berry curvature has the opposite sign along $\overline{\Gamma K}$ and $\overline{\Gamma K}^{\prime}$ due to time-reversal symmetry.

The nontrivial wavefunction on the TSS with scattering by impurities or phonons can give rise to finite electrical $\mathrm{SHG}^{24}$. To investigate the microscopic mechanism, we examine the scaling properties of the second-order transport with respect to the linear conductivity $\sigma$ of the film using the data in Figs. 1d and 2e. Figure 4a shows that the experimental data fit well with $\frac{E_{y}^{(2)}}{E_{x}^{(2)}}=a \sigma^{2}+b$, where $E_{y}^{(2)}=\frac{V_{y}^{2 \omega}}{W}$ and $E_{x}=\frac{V_{x}^{\omega}}{L}(W$ and $L$ are the width and length of the sample, respectively). The linear and second-order conductivities $\sigma$ and $\sigma_{y x x}^{(2)}$ are related by $J_{y}^{(2)}=\sigma_{y x x}^{(2)} E_{x}^{2}=\sigma E_{y}^{(2)}$, so the coefficients $a$ and $b$ represent contributions in $\sigma_{y x x}^{(2)}$ that scale as $\sigma^{3}$ and $\sigma$, respectively. Furthermore, $\sigma$ is proportional to $\tau$ for low frequencies compared to $\tau^{-1}$. Therefore, the intercept $b$ amounts to the $\tau$ linear contributions of the second-order conductivity, which are generally attributed to $\mathrm{BCD}^{3}$ and/or side jump ${ }^{6}$. Note that the former is absent in our case for the symmetry reason, so we attribute the $\tau$-linear contribution to side jump. On the other hand, the slope $a$ quantifies the contribution $\sigma_{y x x}^{(2)} \propto \tau^{3}$, which originates from skew scattering as we discuss below. We obtain similar fitting results for $\Theta=15^{\circ}$ in Fig. $4 \mathrm{~b}$ and also in 10 QL $\mathrm{Bi}_{2} \mathrm{Se}_{3}$ (see Supplementary Fig. 2). Notably, the cubic contribution plays a dominant role over the linear one as $\sigma$ increases in $\mathrm{Bi}_{2} \mathrm{Se}_{3}$, and these two contributions are of opposite signs as shown in Fig. 4a, b and are separated in Supplementary Fig. 3. The scaling of electrical SHG with respect to the surface linear conductivity $\sigma_{s}$ is also analyzed in Supplementary Fig. 4.

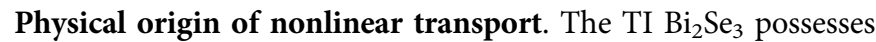
time-reversal and inversion symmetries in the bulk. However, inversion is broken on the surface and hence the metallic TSS with $C_{3 \mathrm{v}}$ crystalline symmetry can host electrical SHG. It takes the form $^{24,34}$

$$
J=\sigma^{(2)}|\mathbf{E}|^{2} \cos 3 \Theta,
$$

where $\Theta$ is the angle of the applied electric field $\mathbf{E}$ with respect to the $\overline{\Gamma K}$ direction and the current density $J$ is measured perpendicular to E. There is only one independent element $\sigma^{(2)}$ in the second-order conductivity tensor $\sigma_{\mathrm{abc}}^{(2)}$ for a two-dimensional system with $C_{3 \mathrm{v}}$ symmetry (see Methods).

Skew scattering is one of the microscopic mechanisms that contributes to $\sigma^{(2)}$. It arises even classically when there are nontrivial impurity potentials lacking inversion on the atomic scale $e^{8,34,35}$ or by local correlation of spins ${ }^{36}$. Alternatively, without relying details of impurities, quantum Bloch functions can imprint inversion breaking and trigger skew scattering, which is the case for the TSS ${ }^{24,34}$. There is a semiclassical picture for skew scattering in a second-order process, schematically depicted in Fig. 4c. The hexagonally warped Fermi surface consists of the positive and negative Berry curvature segments. Since both segments are anisotropic, they acquire finite but opposite velocities in the second-order response. When we construct a wave packet from states on the Fermi surface, it self-rotates due to finite Berry curvature and the rotation direction depends on the sign of Berry curvature. Like the Magnus effect, even an isotropic scatterer deflects the motion of wave packets in a preferred direction due to the self-rotation, thus leading to finite SHG.

The semiclassical Boltzmann transport calculation ${ }^{24}$ based on the model Hamiltonian of TSS ${ }^{32,37}$ leads to the linear conductivity from the TSS $\sigma_{\text {TSS }}=\frac{e^{2} \tau \epsilon_{F}}{4 \pi \hbar^{2}}$ and the second-order conductivity from skew scattering is given by $\sigma^{(2)}=\frac{e^{3} v \tau^{3}}{\hbar^{2} \tau}$, where $\tau$ is the transport scattering time, $\widetilde{\tau}$ is the skew scattering time, $e$ is the electric charge, $\epsilon_{F}$ is the Fermi energy, and $v$ is the Dirac velocity. Importantly, skew scattering yields $\sigma^{(2)} \propto \tau^{3}$ (assuming that $\widetilde{\tau}$ is constant) while other contributions including side jump have weaker powers in $\tau$, which distinguishes the skew scattering 
a

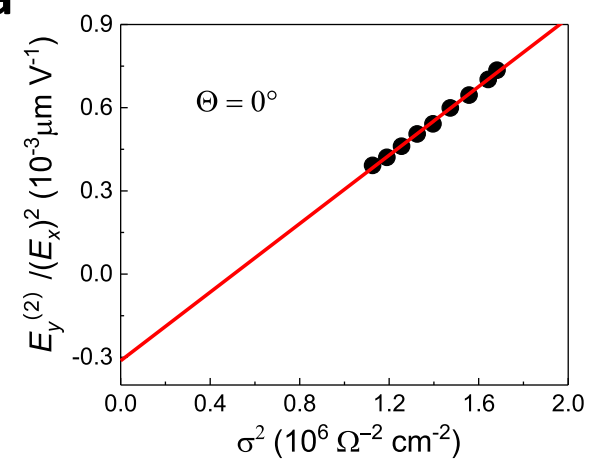

C

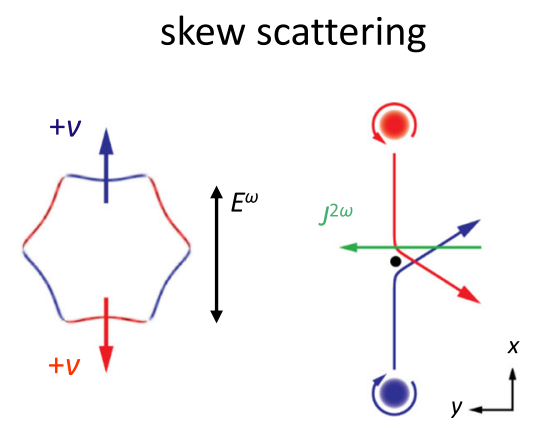

b
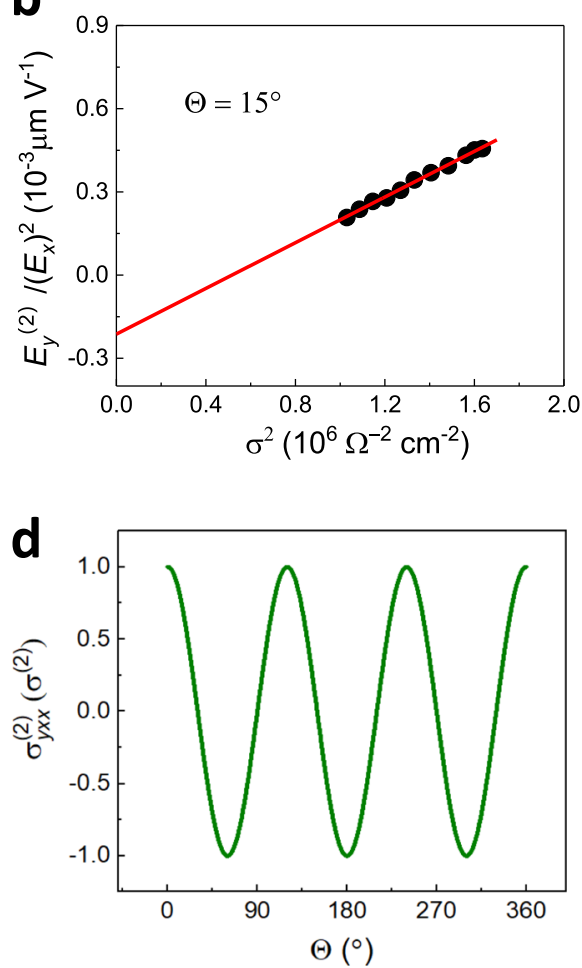

Fig. 4 Physical origin of nonlinear transport. $\mathbf{a}, \mathbf{b} \mathrm{E}_{\mathrm{y}}^{(2)} / \mathrm{E}_{\mathrm{x}}^{2}$ versus the square of the longitudinal conductivity $\left(\sigma^{2}\right)$ for the current injection angles at $\Theta=0^{\circ}$ (a) $15^{\circ}(\mathbf{b})$ in temperature of $50-200 \mathrm{~K}$, where the electron transport is strongly determined by electron-acoustic phonon scattering ${ }^{28}$. The red line is a linear fit to the experimental data. c Schematic plot of the skew scattering of surface Dirac fermion and the induced nonlinear transverse transport. d The theoretical nonlinear transverse conductivity versus the applied electric field direction $\Theta$ based on the skew scattering of TSS.

contribution. The experimentally observed $\sigma_{y x x}^{(2)} \propto \sigma^{3}$ behavior is supported by the skew scattering mechanism, whose contribution is the largest in our observations.

The second-order conductivity obeys the surface crystalline symmetry to have the form $\sigma_{y x x}^{(2)}=\sigma^{(2)} \cos 3 \Theta$, according to Eq. (2) (Fig. 4d), which is in agreement with our experiment. Instead of a BCD, the threefold rotational symmetry inspires us to define the Berry curvature triple T, a higher-order moment of the Berry curvature distribution in the momentum space. It quantifies the strength of the Berry curvature on the Fermi surface, respecting threefold rotation: $T\left(\epsilon_{F}\right)=2 \pi \hbar \int \frac{d^{2} k}{(2 \pi)^{2}} \delta\left(\epsilon_{F}-\epsilon_{\mathbf{k}}\right) \Omega_{z}(\mathbf{k}) \cos 3 \theta_{\mathbf{k}}$ ( $\theta_{\mathrm{k}}$ : the angle measured from the $\overline{\Gamma K}$ line). For the TSS, we obtain $T\left(\epsilon_{F}\right)=\frac{\lambda \epsilon_{F}}{2 \hbar^{2} v^{3}}$. The Berry curvature triple is related to the skew scattering time $\tilde{\tau}$. When we consider unscreened Coulomb impurities with the strength characterized by the dimensionless parameter $\alpha=\frac{e^{2} Q}{4 \pi \varepsilon_{0} \varepsilon \hbar v}$, where $Q$ is the impurity charge, $\varepsilon_{0}$ is the vacuum permittivity, and $\varepsilon$ is the dielectric constant, we find $\widetilde{\tau} \approx 4 \pi^{2} n_{i} \alpha^{3} v^{2} T\left(\epsilon_{F}\right)$ (see Supplementary Note 2 ).

We now provide the theoretical estimate of the second-order response from skew scattering. Though the second-order response arises only on the surface, both $2 \mathrm{D}$ surface and bulk states contribute to $\sigma$. As the contribution from the TSS is $\sim 40 \%$ from the top and bottom surfaces ${ }^{38}$, we estimate $\tau \approx 0.1$ ps and $\tilde{\tau} \approx 10$ ps (see Methods section). The ratio $\tau / \widetilde{\tau}$ of $\sim 1 \%$ quantifies the relative strength of skew scattering. The estimated $\tau$ and $\widetilde{\tau}$ result in the theoretical value $\sigma^{(2)} \approx 1.0 \times 10^{-11} \mathrm{~A} \cdot \mathrm{V}^{-2} \cdot \mathrm{m}$. This is about three times larger than the experimentally observed value $\sigma^{(2)}=2.9 \times 10^{-12} \mathrm{~A} \cdot \mathrm{V}^{-2} \cdot \mathrm{m}$. We can attribute this difference to the partial cancellation of the second-order response; the contribution of the top surface is dominant over that of the bottom surface. In addition, screening of the Coulomb interaction reduces the response (see Supplementary Fig. 5).

\section{Discussion}

We have demonstrated the electric SHG in a nonmagnetic 3D TI under zero magnetic field. It provides an example of BCDindependent nonlinear transverse transport, which is further revealed to arise from skew scattering. This skew scattering mechanism can be applicable to a broader class of noncentrosymmetric quantum materials, utilizing the chirality of electron wavefunction in Weyl and Dirac fermions ${ }^{39}$. Though our work reveals the nonlinear transport under low frequencies, it can be extended to higher frequency regimes such as $\mathrm{GHz}$ and THz. Thus, the electric SHG is complementary to previous optoelectronic approaches 34,40 to reveal the underlying physics of nonlinear effects.

Berry curvature is allowed to exist in the TSS ${ }^{31,41}$, and concentrates in regions around $\bar{K}\left(\bar{K}^{\prime}\right)$ points in Fig. 1b, leading to finite Berry curvature triple. Finite Berry curvature also affects the electron distribution function through the collision integral and the anomalous and side jump velocities ${ }^{24}$. The intrinsic contribution due to the anomalous velocity and hence BCD is absent in $\mathrm{Bi}_{2} \mathrm{Se}_{3}$ due to the symmetry reason ${ }^{3}$; however, the extrinsic contributions such as skew scattering and side jump persist ${ }^{21}$. The skew scattering contribution dominates in the weak impurity limit $(\tau \rightarrow \infty)^{23,24}$ because of its high-order $\tau$ dependence. Though a full quantitative understanding of various contributions to nonlinear electric transports remains elusive ${ }^{21}$ which may include phonons, domain boundaries, impurities, and Berry curvature $^{42}$, identifying major mechanisms is an important step not only for the fundamental understanding of underlying principle, but for the development of rectification or second-harmonic 
devices for energy harvesting and high-frequency communication. The extrinsic nonlinear effect observed in $\mathrm{Bi}_{2} \mathrm{Se}_{3}$ is comparable in magnitude to the intrinsic one in few-layer $\mathrm{WTe}_{2}{ }^{6}$, which has a $2 \mathrm{D}$ nonlinear conductivity of $\sim 10^{-12} \mathrm{~A} \cdot \mathrm{V}^{-2} \cdot \mathrm{m}$. Moreover, the extrinsic mechanism exemplified here applies to a wider class of materials with inversion-symmetry breaking, such as graphene/hexagonal-boron-nitride heterostructures ${ }^{43}$, Dirac semimetal $\mathrm{ZrTe}_{5}{ }^{44,45}$ and the two-dimensional electron gas at the $\mathrm{LaAlO}_{3} / \mathrm{SrTiO}_{3}$ interface ${ }^{46}$. Engineering scattering processes in above materials is a promising way to achieve a prominent SHG by utilizing their much higher carrier mobilities. A higher mobility and long scattering time improve the efficiency in device applications since skew scattering has a higher order dependence on $\tau^{1,24,47}$

\section{Methods}

Sample preparation and electric measurements. $\mathrm{Bi}_{2} \mathrm{Se}_{3}$ films were grown on $\mathrm{Al}_{2} \mathrm{O}_{3}$ (0001) substrates in a molecular beam epitaxy system with a base pressure $<2 \times 10^{-9}$ mbar, as detailed in Tian et al. ${ }^{47}$. Van der Waals epitaxy of $\mathrm{Bi}_{2} \mathrm{Se}_{3}$ film was achieved by adopting the two-step growth method $25,27,48,49$. For transport measurements, a capping layer of $\mathrm{MgO}(2 \mathrm{~nm}) / \mathrm{Al}_{2} \mathrm{O}_{3}(3 \mathrm{~nm})$ was deposited on top of the films prior to device fabrication. Hall bar devices were fabricated using the standard photolithography and Argon plasma etching. They were wire-bonded to the sample holder and installed in a physical property measurement system (PPMS, Quantum Design) for transport measurements. We performed low-frequency ac harmonic electric measurements, using Keithley 6221 current sources and Stanford Research SR830 lock-in amplifiers. During the measurements, a sinusoidal current with a constant amplitude and certain frequency is applied to the devices, and the in-phase first harmonic $V_{\omega}$ and out-of-phase second harmonic $V_{2 \omega}$ longitudinal and transverse voltages were measured simultaneously by four lock-in amplifiers.

Theoretical modeling and estimate. The Hamiltonian for the TSS is ${ }^{32,37}$

$$
H=\hbar v\left(k_{x} \sigma_{y}-k_{y} \sigma_{x}\right)+\frac{\lambda}{2}\left(k_{+}^{3}+k_{-}^{3}\right) \sigma_{z}
$$

where $k_{ \pm}=k_{x} \pm i k_{y}, \sigma_{a}$ denotes the Pauli matrix $(a=x, y, z)$, and $\lambda$ quantifies the hexagonal warping ${ }^{32}$. In this section, the $x$ axis is set perpendicular to the reflection plane, i.e., along the $\overline{\Gamma K}$ line. For the surface state of $\mathrm{Bi}_{2} \mathrm{Se}_{3}$, we find $v=5 \times 10^{5} \mathrm{~m} / \mathrm{s}$ and $\lambda=80 \mathrm{eV} \cdot \AA^{3}$, and the FS is located above the Dirac point, where a hexagonally warped FS was found 30,33

In general, the current response quadratic to the electric field $E$ takes the form $J_{a}^{(2)}=\sigma_{a b c}^{(2)} E_{b} E_{c}$, where $\sigma_{a b c}^{(2)}$ is the second-order conductivity. For a twodimensional system with $C_{3 \mathrm{~V}}$ symmetry like the TSS, it has only one independent element $\sigma^{(2)} \equiv \sigma_{x x y}^{(2)}=\sigma_{x y x}^{(2)}=\sigma_{y x x}^{(2)}=-\sigma_{y y y}^{(2)}$. To estimate the transport properties, we assume Coulomb impurities, randomly distributed in a sample. Taking account of the Thomas-Fermi screening, we write the Fourier transform of the Coulomb interaction as $V(q)=\frac{2 \pi \alpha \hbar v}{q+q_{\mathrm{TF}}}$, where $q_{\mathrm{TF}}$ is the Thomas-Fermi wavevector. Here, we consider unscreened Coulomb impurities $\left(q_{\mathrm{TF}}=0\right)$, which we discuss below.

In estimating $\tau$ and $\tilde{\tau}$, we use the dielectric constant ${ }^{50} \in \approx 100$, leading to $\alpha \approx \frac{1}{23}$. We use the previous observation that the contribution of the TSS from the top and bottom surfaces to the total conduction is $\sim 40 \%{ }^{38}$ and assume that the impurity density $n_{i}$ is approximately the same as the carrier density $n_{2 \mathrm{D}}$. Thus, the observed linear conductivity $\sigma=2.5 \times 10^{-3} \Omega^{-1}$ at $10 \mathrm{~K}$ leads to the carrier density of the TSS $n_{\text {TSS }}=2.43 \times 10^{12} \mathrm{~cm}^{-2}$, the corresponding Fermi wavelength

$\lambda_{F}=\sqrt{\frac{\pi}{n_{\mathrm{TSS}}}}=11.4 \mathrm{~nm}$, the scattering time $\tau \approx 0.1 \mathrm{ps}$, and the skew scattering time $\tilde{\tau} \approx 10 \mathrm{ps}$, where we use the expressions ${ }^{24} \sigma_{\mathrm{TSS}}=\frac{e^{2} \tau \epsilon_{F}}{4 \pi \hbar^{2}}, \tau^{-1}=\frac{\pi}{2} n_{i} \alpha^{2} v \lambda_{F}$, and $\tilde{\tau}^{-1}=\frac{4 \pi^{3}}{\hbar} \frac{n_{i} \alpha^{3} \lambda}{\lambda_{F}}$. The small ratio of $\frac{\tau}{\tau} \ll 1$ satisfies the condition of the perturbative treatment of impurities in the semiclassical Boltzmann theory.

The Thomas-Fermi wavelength $\lambda_{\mathrm{TF}}=\frac{2 \pi}{q_{\mathrm{TF}}}$ is typically ranging from 26 to 90 $\mathrm{nm}^{51,52}$, resulting in the ratio $\lambda_{F} / \lambda_{\mathrm{TF}} \lesssim 0.4$. We describe the detailed calculations and discussion about the effect of screening in Supplementary Note 2 and Supplementary Fig. 5. We note that for short-range impurities or in the strong screening limit, i.e., $\lambda_{\mathrm{TF}} \rightarrow 0$, skew scattering vanishes in a gapless Dirac system $^{24,34}$.

\section{Data availability}

The data that support the plots within this paper and other findings of this study are available from the corresponding author upon reasonable request.

Received: 8 July 2020; Accepted: 6 January 2021;

Published online: 29 January 2021

\section{References}

1. Nagaosa, N., Sinova, J., Onoda, S., MacDonald, A. H. \& Ong, N. P. Anomalous Hall effect. Rev. Mod. Phys. 82, 1539-1592 (2010).

2. Gao, Y., Yang, S. A. \& Niu, Q. Field induced positional shift of bloch electrons and its dynamical implications. Phys. Rev. Lett. 112, 166601 (2014).

3. Sodemann, I. \& Fu, L. Quantum nonlinear hall effect induced by berry curvature dipole in time-reversal invariant materials. Phys. Rev. Lett. 115, 216806 (2015)

4. Hamamoto, K., Ezawa, M., Kim, K. W., Morimoto, T. \& Nagaosa, N. Nonlinear spin current generation in noncentrosymmetric spin-orbit coupled systems. Phys. Rev. B 95, 224430 (2017).

5. Ma, Q. et al. Observation of the nonlinear Hall effect under time-reversalsymmetric conditions. Nature 565, 337-342 (2019).

6. Kang, K., Li, T., Sohn, E., Shan, J. \& Mak, K. F. Nonlinear anomalous Hall effect in few-layer WTe $\mathrm{W}_{2}$. Nat. Mater. 18, 324-328 (2019).

7. Shen, Y.-R. The Principles Of Nonlinear Optics (Wiley-Interscience, New York, NY, 1984)

8. Belinicher, V. I. \& Sturman, B. I. The photogalvanic effect in media lacking a center of symmetry. Sov. Phys. Uspekhi 23, 199-223 (1980).

9. Moore, J. E. \& Orenstein, J. Confinement-induced berry phase and helicitydependent photocurrents. Phys. Rev. Lett. 105, 026805 (2010).

10. Du, Z. Z., Wang, C. M., Lu, H.-Z. \& Xie, X. C. Band signatures for strong nonlinear hall effect in bilayer WTe $\mathrm{W}_{2}$. Phys. Rev. Lett. 121, 266601 (2018).

11. Zhang, Y., van den Brink, J., Felser, C. \& Yan, B. Electrically tuneable nonlinear anomalous Hall effect in two-dimensional transition-metal dichalcogenides $\mathrm{WTe}_{2}$ and $\mathrm{MoTe}_{2}$. 2D Materials 5, 044001 (2018).

12. Facio, J. I. et al. Strongly enhanced berry dipole at topological phase transitions in BiTeI. Phys. Rev. Lett. 121, 246403 (2018).

13. You, J.-S., Fang, S., Xu, S.-Y., Kaxiras, E. \& Low, T. Berry curvature dipole current in the transition metal dichalcogenides family. Phys. Rev. B 98, 121109 (2018).

14. Zhang, Y., Sun, Y. \& Yan, B. Berry curvature dipole in Weyl semimetal materials: an ab initio study. Phys. Rev. B 97, 041101 (2018).

15. Hsieh, D. et al. Observation of unconventional quantum spin textures in topological insulators. Science 323, 919-922 (2009).

16. Chen, Y. L. et al. Experimental realization of a three-dimensional topological insulator, $\mathrm{Bi}_{2} \mathrm{Te}_{3}$. Science 325, 178-181 (2009).

17. Hasan, M. Z. \& Kane, C. L. Colloquium. Rev. Mod. Phys. 82, 3045-3067 (2010).

18. Yazyev, O. V., Moore, J. E. \& Louie, S. G. Spin polarization and transport of surface states in the topological insulators $\mathrm{Bi}_{2} \mathrm{Se}_{3}$ and $\mathrm{Bi}_{2} \mathrm{Te}_{3}$ from first principles. Phys. Rev. Lett. 105, 266806 (2010).

19. Burkov, A. A. \& Hawthorn, D. G. Spin and charge transport on the surface of a topological insulator. Phys. Rev. Lett. 105, 066802 (2010).

20. Moore, J. E. The birth of topological insulators. Nature 464, 194 (2010).

21. Du, Z. Z., Wang, C. M., Li, S., Lu, H.-Z. \& Xie, X. C. Disorder-induced nonlinear Hall effect with time-reversal symmetry. Nat. Commun. 10, 3047 (2019).

22. König, E. J., Dzero, M., Levchenko, A. \& Pesin, D. A. Gyrotropic Hall effect in Berry-curved materials. Phys. Rev. B 99, 155404 (2019).

23. Nandy, S. \& Sodemann, I. Symmetry and quantum kinetics of the nonlinear Hall effect. Phys. Rev. B 100, 195117 (2019).

24. Isobe, H., Xu, S.-Y. \& Fu, L. High-frequency rectification via chiral Bloch electrons. Sci. Adv. 6, eaay2497 (2020).

25. Bansal, N. et al. Epitaxial growth of topological insulator $\mathrm{Bi}_{2} \mathrm{Se}_{3}$ film on $\mathrm{Si}$ (111) with atomically sharp interface. Thin Solid Films 520, 224-229 (2011).

26. Lee, J., Wang, Z., Xie, H., Mak, K. F. \& Shan, J. Valley magnetoelectricity in single-layer $\mathrm{MoS}_{2}$. Nat. Mater. 16, 887 (2017).

27. Bansal, N., Kim, Y. S., Brahlek, M., Edrey, E. \& Oh, S. Thickness-independent transport channels in topological insulator $\mathrm{Bi}_{2} \mathrm{Se}_{3}$ thin films. Phys. Rev. Lett. 109, 116804 (2012).

28. Kim, D. et al. Intrinsic electron-phonon resistivity of $\mathrm{Bi}_{2} \mathrm{Se}_{3}$ in the topological regime. Phys. Rev. Lett. 109, 166801 (2012).

29. Chen, J. et al. Gate-voltage control of chemical potential and weak antilocalization in $\mathrm{Bi}_{2} \mathrm{Se}_{3}$. Phys. Rev. Lett. 105, 176602 (2010).

30. Wang, Y. H. et al. Observation of a warped helical spin texture in $\mathrm{Bi}_{2} \mathrm{Se}_{3}$ from circular dichroism angle-resolved photoemission spectroscopy. Phys. Rev. Lett. 107, 207602 (2011).

31. Li, Z. \& Carbotte, J. P. Hexagonal warping on spin texture, Hall conductivity, and circular dichroism of topological insulators. Phys. Rev. B 89, 165420 (2014).

32. $\mathrm{Fu}, \mathrm{L}$. Hexagonal warping effects in the surface states of the topological insulator $\mathrm{Bi}_{2} \mathrm{Te}_{3}$. Phys. Rev. Lett. 103, 266801 (2009).

33. Kuroda, K. et al. Hexagonally deformed fermi surface of the $3 \mathrm{D}$ topological insulator $\mathrm{Bi}_{2} \mathrm{Se}_{3}$. Phys. Rev. Lett. 105, 076802 (2010).

34. Olbrich, P. et al. Room-temperature high-frequency transport of dirac fermions in epitaxially grown $\mathrm{Sb}_{2} \mathrm{Te}_{3}$ - and $\mathrm{Bi}_{2} \mathrm{Te}_{3}$-based topological insulators. Phys. Rev. Lett. 113, 096601 (2014).

35. Sinitsyn, N. A., Niu, Q. \& MacDonald, A. H. Coordinate shift in the semiclassical Boltzmann equation and the anomalous Hall effect. Phys. Rev. B 73, 075318 (2006). 
36. Ishizuka, H. \& Nagaosa, N. Spin chirality induced skew scattering and anomalous Hall effect in chiral magnets. Sci. Adv. 4, eaap9962 (2018).

37. Liu, C.-X. et al. Model Hamiltonian for topological insulators. Phys. Rev. B 82, 045122 (2010)

38. Wang, Y. et al. Room temperature magnetization switching in topological insulator-ferromagnet heterostructures by spin-orbit torques. Nat. Commun. 8, 1364 (2017)

39. Armitage, N. P., Mele, E. J. \& Vishwanath, A. Weyl and Dirac semimetals in three-dimensional solids. Rev. Mod. Phys. 90, 015001 (2018)

40. Soifer, H. et al. Band-resolved imaging of photocurrent in a topological insulator. Phys. Rev. Lett. 122, 167401 (2019).

41. Hosur, P. Circular photogalvanic effect on topological insulator surfaces: Berry-curvature-dependent response. Phys. Rev. B 83, 035309 (2011).

42. Ishizuka, H. \& Nagaosa, N. Noncommutative quantum mechanics and skew scattering in ferromagnetic metals. Phys. Rev. B 96, 165202 (2017).

43. Dean, C. R. et al. Boron nitride substrates for high-quality graphene electronics. Nat. Nanotechnol. 5, 722 (2010).

44. Li, Q. et al. Chiral magnetic effect in $\mathrm{ZrTe}_{5}$. Nat. Phys. 12, 550 (2016).

45. Liu, Y. et al. Zeeman splitting and dynamical mass generation in Dirac

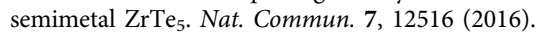

46. Ohtomo, A. \& Hwang, H. Y. A high-mobility electron gas at the $\mathrm{LaAlO}_{3} /$ $\mathrm{SrTiO}_{3}$ heterointerface. Nature 427, 423-426 (2004).

47. Tian, Y., Ye, L. \& Jin, X. Proper scaling of the anomalous Hall Effect. Phys Rev. Lett. 103, 087206 (2009).

48. $\mathrm{Li}, \mathrm{H}$. D. et al. The van der Waals epitaxy of $\mathrm{Bi}_{2} \mathrm{Se}_{3}$ on the vicinal $\mathrm{Si}(111)$ surface: an approach for preparing high-quality thin films of a topological insulator. New J. Phys. 12, 103038 (2010).

49. He, P. et al. Bilinear magnetoelectric resistance as a probe of threedimensional spin texture in topological surface states. Nat. Phys. 14, 495-499 (2018).

50. Kim, D. et al. Surface conduction of topological Dirac electrons in bulk insulating $\mathrm{Bi}_{2} \mathrm{Se}_{3}$. Nat. Phys. 8, 459-463 (2012).

51. Beidenkopf, H. et al. Spatial fluctuations of helical Dirac fermions on the surface of topological insulators. Nat. Phys. 7, 939-943 (2011).

52. Foster, M. S. Multifractal nature of the surface local density of states in threedimensional topological insulators with magnetic and nonmagnetic disorder. Phys. Rev. B 85, 085122 (2012).

\section{Acknowledgements}

We thank Steven S.-L. Zhang and G. Vignale for discussions. The work was partially supported by SpOT-LITE program (A*STAR grant, A18A6b0057) through RIE2020 funds, and Singapore Ministry of Education (MOE) Tier 1 (R263-000-D61-114). P.H. acknowledges the start-up funding from Fudan University.

\section{Author contributions}

P.H. fabricated the devices, performed transport measurements, and analyzed the data. D.Z. grew the films. H.I. and L.F. performed theoretical studies. C.-H.H. contributed to Berry curvature calculation. All authors discussed the results. P.H., H.I., L.F., and H.Y. wrote the manuscript.

\section{Competing interests}

The authors declare no competing interests.

\section{Additional information}

Supplementary informationThe online version contains supplementary material available at https://doi.org/10.1038/s41467-021-20983-1.

Correspondence and requests for materials should be addressed to L.F. or H.Y.

Peer review information Nature Communications thanks Hua Jiang and the other, anonymous, reviewer(s) for their contribution to the peer review of this work.

Reprints and permission information is available at http://www.nature.com/reprints

Publisher's note Springer Nature remains neutral with regard to jurisdictional claims in published maps and institutional affiliations.

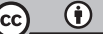

Open Access This article is licensed under a Creative Commons Attribution 4.0 International License, which permits use, sharing, adaptation, distribution and reproduction in any medium or format, as long as you give appropriate credit to the original author(s) and the source, provide a link to the Creative Commons license, and indicate if changes were made. The images or other third party material in this article are included in the article's Creative Commons license, unless indicated otherwise in a credit line to the material. If material is not included in the article's Creative Commons license and your intended use is not permitted by statutory regulation or exceeds the permitted use, you will need to obtain permission directly from the copyright holder. To view a copy of this license, visit http://creativecommons.org/ licenses/by/4.0/

(C) The Author(s) 2021 